\title{
Efecto de la azitromicina en la producción de biopelículas y la composición de Acinetobacter baumannii resistente a múltiples fárrmacos
}

\author{
Adrián Camacho-Ortiz ${ }^{*}$, Reynaldo Lara-Medrano, Michel F. Martínez-Reséndez", \\ Soraya Mendoza-Olazarán², Samantha Flores-Treviño y Elvira Garza-González² \\ ${ }^{1}$ Servicio de Infectología; ${ }^{2}$ Servicio de Gastroenterología. Universidad Autónoma de Nuevo León, Hospital Universitario Dr. José E. González y \\ Facultad de Medicina, Monterrey, N.L., México
}

\section{Resumen}

Objetivos: Evaluar el efecto de la azitromicina (AZM) en la formación y composición de biopelículas en Acinetobacter baumannii resistente a múltiples fármacos (MDR). Material y métodos: Se estudiaron 96 aislamientos de A. baumannii. La susceptibilidad antimicrobiana y la concentración inhibitoria submínima (sub-MIC) se determinaron por el método de microdilución del caldo. Los genes carbapenemasa fueron detectados por reacción en cadena de la polimerasa y la diversidad clonal por electroforesis en gel de campos pulsados (PFGE). La formación de biopelículas sin AZM y la sub-MIC de AZM por tinción de cristal violeta. La composición de la biopelícula sin AZM y la sub-MIC de AZM se determinaron mediante ensayos de desprendimiento. Resultados: Los A. baumannii seleccionados fueron MDR; el 93.8\% resistentes al carbapenem y 24 OXA-24 positivos. El PFGE demostró predominancia en los clones A (53\%), B (34.7\%) y C (12.5\%). La producción de biopelículas en sub-MIC de AZM disminuyó en un 53.1\%, aumentó en un 34.7\% y no mostró diferencias en un $12.5 \%$ de Ios aislamientos, comparado con la producción de biopelículas sin AZM. Conclusión: La sub-MIC de AZM puede reducir la producción de biopelículas en aislamientos de A. baumannii MDR con disminución de proteínas y el ADN en la biopelícula. Nuestros resultados pueden ser útiles en estudios de sinergia para nuevas alternativas terapéuticas.

PALABRAS CLAVE: Acinetobacter baumannii. Biopelícula. Sub-MIC. Composición de biopelícula.

\section{Azithromycin effect on multidrug-resistant Acinetobacter baumannii biofilm production and composition}

\begin{abstract}
Objectives: To evaluate the effect of azithromycin (AZM) on biofilm formation and composition in multidrug resistant (MDR) Acinetobacter baumannii. Material and methods: Ninety-six A. baumannii isolates were studied. Antimicrobial susceptibility and sub-minimum inhibitory concentration (sub-MIC) were determined by the broth microdilution method. Carbapenemase genes were detected by polymerase chain reaction and clonal diversity by pulsed-field gel electrophoresis (PFGE). Biofilm formation without AZM and AZM sub-MIC were determined by crystal violet staining. AZM-free biofilm composition and AZM sub-MIC were determined by detachment assays. Results: The selected A. baumannii were MDR; $93.8 \%$ were carbapenem-resistant and 24 were OXA-24-positive. PFGE showed predominance of clones $A$ (53\%), B (34.7\%) and C (12.5\%). Biofilm production at AZM sub-MICs decreased in $53.1 \%$, increased in $34.7 \%$ and showed no differences in $12.5 \%$ of isolates, in comparison with biofilm production without AZM. Conclusion: AZM sub-MIC can reduce biofilm production in A. baumannii MDR isolates with decreased protein and DNA in the biofilm. Our results may be useful in synergy studies for new therapeutic alternatives.
\end{abstract}

KEY WORDS: Acinetobacter baumannii. Biofilm. Sub-MIC. Biofilm composition

Correspondencia:

*Adrián Camacho Ortiz

E-mail: acamacho_md@yahoo.com

\section{Fecha de recepción: 16-06-2021}

Fecha de aceptación: 13-07-2021

DOI: $10.24875 /$ GMM.21000380
Gac Med Mex. 2021;157:497-502

Disponible en PubMed

www.gacetamedicademexico.com

0016-3813/@ 2021 Academia Nacional de Medicina de México, A.C. Publicado por Permanyer. Este es un artículo open access bajo la licencia CC BY-NC-ND (http://creativecommons.org/licenses/by-nc-nd/4.0/). 


\section{Introducción}

Acinetobacter baumannii es un cocobacilo gramnegativo oportunista asociado a los brotes en las unidades de cuidados intensivos (UCI) ${ }^{1}$. Esta especie está relacionada con neumonías asociadas a ventilador, bacteriemia, infecciones de sitio quirúrgico y de vías urinarias, siendo las dos primeras las más importantes debido a su alta morbilidad y mortalidad asociada ${ }^{1,2}$.

La resistencia antibiótica en las infecciones nosocomiales de $A$. baumannii es una amenaza de salud mundial ${ }^{2,3}$, y es por esta razón que esta especie está en la lista de patógenos resistentes a los antibióticos con una prioridad mundial para la investigación y el desarrollo de nuevos y efectivos tratamientos antibióticos ${ }^{4}$.

Uno de los factores de virulencia más importantes de $A$. baumannii es la producción de biopelículas, las cuales protegen al microrganismo del sistema inmunitario del huésped y del tratamiento con antibióticos ${ }^{5,6}$.

La azitromicina (AZM) es un antibiótico macrólido que inhibe la síntesis de proteínas bacterianas y está indicado en infecciones respiratorias, urogenitales, dérmicas y otras infecciones bacterianas causadas principalmente por especies de bacterias grampositivas $^{7}$. En las especies gramnegativas se ha demostrado que este antibiótico disminuye la producción de biopelículas, por ejemplo en Pseudomonas aeruginosa ${ }^{8}$, Stenotrophomonas maltophilia ${ }^{9}$, Porphyromonas gingivalis ${ }^{10}$ y Haemophilus influenzae.

De acuerdo con la literatura, el efecto de la AZM en las células del biopelículas de $A$. baumannii no ha sido evaluado en detalle. Por lo tanto, el objetivo de este estudio fue evaluar el efecto de la AZM en la formación de biopelículas en los aislamientos clínicos de A. baumannii.

\section{Materiales y métodos}

\section{Aislamiento de sitios y clínicas}

El estudio se llevó a cabo en el Hospital Universitario Dr. José Eleuterio González, un hospital de atención terciaria en Monterrey, México. De enero a diciembre de 2012 se recuperaron un total de 149 aislamientos clínicos de $A$. baumannii de pacientes de la UCl. Solo se incluyó un aislamiento por paciente. Los aislamientos de $A$. baumannii se identificaron mediante la espectrometría de masas MALDI-TOF (Bruker
Daltonics, Bremen) y mediante la amplificación del gen espaciador intergénico por reacción en cadena de la polimerasa $(\mathrm{PCR})^{11}$. Todos los aislamientos fueron almacenados hasta su uso a $-70{ }^{\circ} \mathrm{C}$ en caldo Brucella con un $15 \%$ de glicerol.

\section{Caracterización y selección de los aislados}

\section{SUSCEPTIBILIDAD ANTIMICROBIANA}

La prueba de susceptibilidad a antibióticos se determinó utilizando el método de microdilución del caldo. Entre los antibióticos examinados se encuentran: amikacina, cefepima, ceftazidima, ciprofloxacino, gentamicina, imipenem, levofloxacino, meropenem y tobramicina (Sigma-Aldrich, Toluca, México). Los resultados se interpretaron de acuerdo con los criterios del Instituto de Estándares Clínicos y de Laboratorio (CLSI) (2018) ${ }^{12}$.

La resistencia a múltiples fármacos (MDR) se definió como la resistencia a por lo menos tres clases diferentes de antibióticos ${ }^{13}$. Solo se seleccionaron aislamientos de MDR en los siguientes ensayos.

\section{Caracterización de LOS GENES DE LA CARBAPENEMASA}

El ADN de la plantilla se preparó por lisis térmica a $95{ }^{\circ} \mathrm{C}$ durante $10 \mathrm{~min}$. Los genes de la carbapenemasa que codifican las metalo- $\beta$-lactamasas, blaVIM, blalMP, y blaNDM, y la carbapenemasa de clase D tipo bla OXA-48 (OXA-23, OXA-24, OXA-51, y OXA-58 $)^{14}$ fueron tipificados como se informó anteriormente ${ }^{15-18}$. En el caso de los genes positivos, los productos de PCR fueron purificados, secuenciados por el método de terminación de cadena con el kit BigDye Terminator (Applied Biosystems, Foster City, CA), $y$ analizados en un analizador genético ABIPRrism 3100 (Applied Biosystems).

\section{Caracterización de La diversidad CLONAL}

Los aislamientos de A. baumannii fueron tipificados por electroforesis en el gel de campos pulsados (PFGE $^{19,20}$. Las muestras de ADN de las cepas fueron digeridas con $10 \mathrm{U}$ de Smal (Takara Bio Inc., Shiga, Japón). EI PFGE se realizó con un instrumento CHEFDRIII (BioRad Laboratories, Hercules, CA), y los patrones de banda fueron interpretados usando el método descrito por Seifert, et al. ${ }^{17}$. Aislamientos con 
patrones de restricción de banda 100\% similares fueron clasificados como pertenecientes al mismo clon.

\section{ProducCIÓn de BIOPELÍCULA}

La producción semicuantitativa de biopelículas se realizó mediante el método de tinción de cristal violeta, como se ha descrito anteriormente ${ }^{21}$. Para el análisis cuantitativo se determinó el índice de biopelícula (IB) para compensar las diferencias en la tasa de crecimiento $^{19}$. Todos los aislamientos fueron probados en cuadruplicados en dos experimentos independientes. Se utilizaron como organismos de control Staphylococcus saprophyticus ATCC 15305 (productor de biopelícula) y el Staphylococcus hominis ATCC 27844 (no productor de biopelícula). Los cortes propuestos por Christensen, et al. se usaron para clasificar los aislamientos. Solo los aislamientos fuertemente productores de biopelícula ( $O D \geq 0.24$ ) fueron seleccionados en los siguientes ensayos.

\section{CONCENTRACIONES MÍNIMAS SUBINHIBITORIAS DE $A Z M$}

Se determinaron las concentraciones mínimas inhibitorias (MIC) de AZM (Sigma-Aldrich, Toluca, México) para ocho aislados de $A$. baumannii seleccionados al azar. Posteriormente, se prepararon diluciones dobles de 0.125 a $8.8 \mu \mathrm{g} / \mathrm{ml}$ y se utilizó un cultivo de $24 \mathrm{~h}$ como inóculo. Después de la incubación a $37^{\circ} \mathrm{C}$, se midió la densidad óptica a $600 \mathrm{~nm}$ (OD 600). Las concentraciones mínimas subinhibitorias (sub-MIC) de AZM se definieron como la concentración en la que se observó un crecimiento inhibido.

\section{EFECTO DE LAS SUBCAPITALIZACIONES DE AZM EN LA PRODUCCIÓN Y COMPOSICIÓN DE BIOPELÍCULAS}

Para el análisis del impacto de la sub-MIC de la AZM en la producción de biopelículas, cada biopelícula madura se cultivó en caldo de soya tríptico suplementado con un $1 \%$ de glucosa sin AZM y con una sub-MIC de AZM durante $24 \mathrm{~h}$. El IB se determinó como se describe ${ }^{19}$. Para el análisis de la sub-MIC de AZM sobre la composición de la biopelícula, se realizaron ensayos de desprendimiento utilizando meta-periodato de sodio (NalO4) para degradar $\beta-1$, polisacáridos de 6 enlaces, proteinasa $\mathrm{K}$ para degradar proteínas, y DNasa I para degradar el DNA como se ha descrito anteriormente ${ }^{22}$. El porcentaje de separación se calculó por la diferencia media entre los pozos tratados y los no tratados. Los ensayos se realizaron sin AZM y con una sub-MIC de AZM.

\section{Análisis estadísticos}

Los datos se analizaron con el programa estadístico SPSS version 20.0 (IBM Corporation, Somers, NY). Los coeficientes de similitud de los patrones de banda del PFGE se generaron a partir de una matriz de similitud calculada con el coeficiente de Jaccard. Se utilizó un análisis descriptivo para las variables cuantitativas, y las variables cualitativas se expresaron como proporciones. La prueba t de Student se utilizó para comparar las muestras emparejadas (índice de biopelícula y porcentaje de desprendimiento de biopelícula). El valor $p<0.05$ se consideró estadísticamente significativo.

\section{Declaración de ética}

Este estudio se realizó con la aprobación del Comité de Ética de la Facultad de Medicina de la Universidad Autónoma de Nuevo León (aprobación n. ${ }^{\circ}$ PII5-0243). Debido a que la información de los pacientes fue anónima, solo se analizaron los datos microbiológicos. Por lo tanto, el comité de ética local no exigía el consentimiento informado.

\section{Resultados}

\section{Aislamientos clínicos, producción de biopelículas y susceptibilidad antimicrobiana}

De un total de 149 cepas de $A$. baumannii recogidas en la UCl, 140 (97\%) resultaron ser fuertes productoras de biopelículas y las 9 restantes $(3 \%)$ fueron productoras débiles.

Todos los aislados fueron resistentes a ciprofloxacino, gentamicina y ceftazidima y el $99 \%$ resistentes a tobramicina y levofloxacino. De todos los aislamientos, el $93.8 \%$ fueron resistentes a los carbapenémicos (meropenem e imipenem), y casi el 70\% resultaron resistentes a cefepima con el $22.9 \%$.

Seleccionamos 96 aislamientos clínicos de $A$. baumannii MDR productor de biopelícula para su posterior análisis; el $80 \%$ se obtuvo de muestras respiratorias y un $12.5 \%$ de hemocultivos, mientras que el resto se obtuvo de otros especímenes. 


\section{Caracterización de los genes de la carbapenemasa y la diversidad clonal}

Los 96 A. baumannii seleccionados resultaron positivos para el gen de la carbapenemasa de tipo OXA, siendo OXA-51 y OXA-24 los más frecuentes con un $100 \%$ y un $96 \%$, respectivamente; ninguno de los aislamientos codificados para los genes de la blaVIM, blalMP, y blaNDM.

Se detectaron doce patrones de PFGE (A-M) con 51 (53\%) identificadas como clon A, 15 (15\%) como clon B y $9(9 \%)$ como clon C.

\section{El efecto de la azitromicina en la producción y composición de la biopelícula}

La sub-MIC seleccionada fue de $0.125125 \mu \mathrm{g} / \mathrm{ml}$. Cuando se evaluaron los 96 aislamientos, la media de IB sin AZM fue de 1.737, y cuando se expuso a la sub-MIC de AZM, el IB fue de 1.488.

Cuando comparamos el IB sin AZM y en la sub-MIC de AZM, la distribución del IB fue heterogénea, con un $53.1 \%$ que reveló una disminución en el IB ( $p<0.0001$ ), un $34.7 \%$ que demostró un aumento en el IB ( $p<0.0001)$, y un $12.5 \%$ que no mostró ninguna diferencia (Fig. 1).

La biopelícula en ausencia de AZM estaba compuesta principalmente de proteínas y DNA (desprendimiento con la proteinasa K, $88 \%$ y la DNasa I, $87 \%$ ), y de carbohidratos (desprendimiento con el meta-periodato, $30 \%)$.

La biopelícula en la sub-MIC de AZM mostró resultados similares (desprendimiento con la proteinasa $\mathrm{K}$, $79 \%$; DNasa I, $77 \%$; y meta-periodato, $25 \%$ ). La proporción de proteínas en el ADN era menor en la biopelícula con la sub-MIC de AZM que en la biopelícula $\sin$ AZM $(p<0.05)$ (Fig. 2).

\section{Discusión}

Uno de los factores de virulencia más importantes de $A$. baumannii es la producción de biopelícula, y se ha demostrado que la AZM inhibe la formación de biopelícula en varias especies bacterianas. Por lo tanto, evaluamos el efecto de la AZM en los aislamientos clínicos de $A$. baumannii MDR y encontramos que cuando se expone a la sub-MIC de la AZM, la producción de biopelículas disminuye con la reducción de la proporción de proteínas en el
ADN. Se ha informado que la AZM disminuye significativamente la producción de biopelícula cuando se utilizan cepas de $A$. baumannii de biopelícula leve y moderada y una subcapa de $1 \mu \mathrm{g} / \mathrm{ml}^{23}$. En este estudio, definimos $0.125 \mu \mathrm{g} / \mathrm{ml}$ como sub-MIC de AZM, y solo se incluyeron cepas fuertemente productoras de biopelícula. El diferencia observada en los valores para el AZM sub-MIC puede deberse a las diferencias en las cepas clínicas utilizadas para este estudio, especialmente por las diferentes producciones de biopelículas. La exposición de las bacterias a la sub-MIC de los antibióticos en condiciones clínicas es muy probable. Algunos ejemplos son: a) por la interacción de los medicamentos, b) por deficiencias en la absorción o c) inmediatamente antes de las siguientes dosis. Por esta razón, es importante señalar que la concentración de $0.125 \mu \mathrm{g} / \mathrm{ml}$ utilizada en nuestros ensayos está por debajo del nivel sérico máximo de AZM cuando se utiliza en dosis intravenosas estándar (nivel sérico máximo de $3.6 \mu \mathrm{g} / \mathrm{ml}$; una dosis de $500 \mathrm{mg}$ IV q24h) $)^{24}$; por lo tanto, esta concentración puede tener un efecto in vivo.

De acuerdo con el CLSI, una concentración subMIC es la concentración de un agente antimicrobiano que no es activa en el crecimiento bacteriano pero que sigue siendo activa en la alteración de la bioquímica y la forma de las bacterias in vitro e in vivo, reduciendo así la virulencia bacteriana. Aunque estas concentraciones sub-MIC no matan a las bacterias, son capaces de modificar sus características y, según nuestros resultados, la composición de la biopelícula está alterada, con una proporción menor de proteínas. Esto puede tener un impacto en importantes funciones celulares bacterianas 0 en la virulencia.

La biopelícula sin AZM está compuesta principalmente por proteínas y ADN, y la biopelícula con la submicroma de AZM mostró una proporción menor de proteínas, lo que puede reflejar la acción de la AZM sobre la síntesis de proteínas. Cabe mencionar que las cepas siguieron siendo fuertes productoras de biopelícula incluso cuando se expusieron a la subMIC de la AZM.

En nuestra institución se ha informado de la distribución clonal de $A$. baumannii a lo largo de seis años $y$, según este estudio, existe una alta clonalidad en esta población ${ }^{25}$.

Para este estudio, la mitad de los aislamientos de $A$. baumannii se agruparon en un clon predominante (A), y los aislamientos restantes se clasificaron en 11 


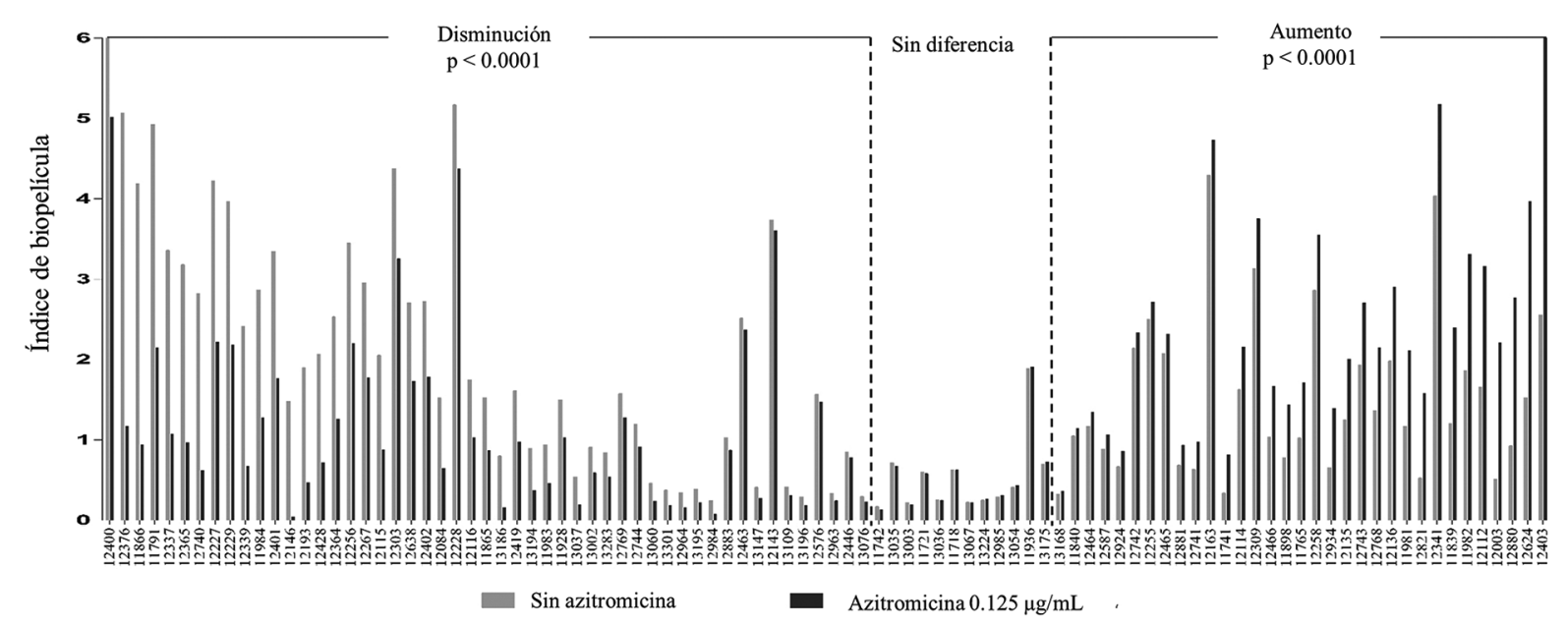

Figura 1. Efecto de la azitromicina en el índice de biopelícula.

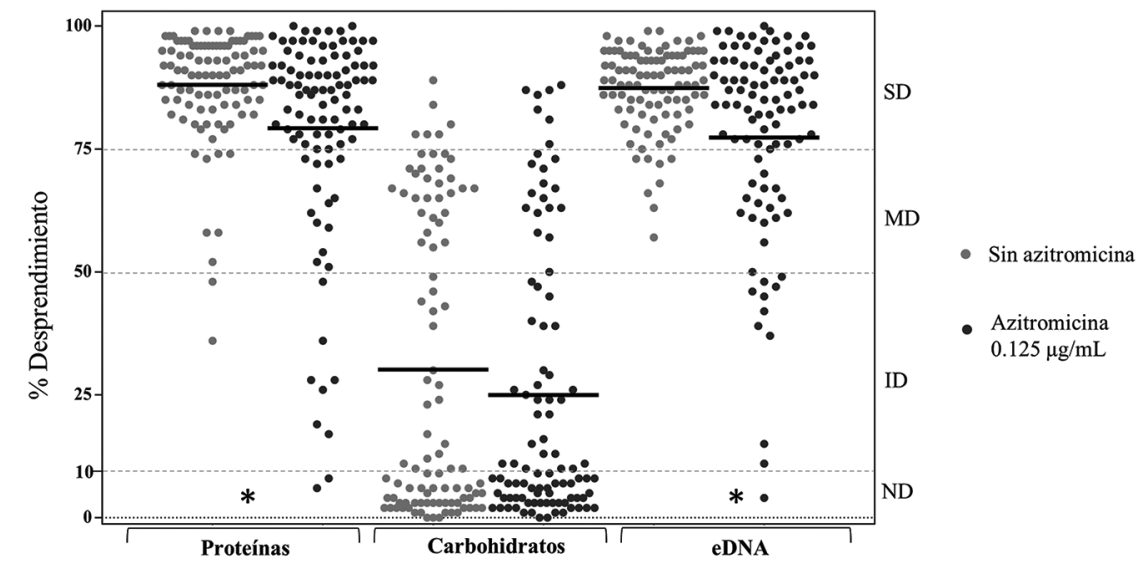

Figura 2. El efecto de la azitromicina en la composición de la biopelícula. ${ }^{*} p<0.001$.

SD: desprendimiento fuerte; MD: desprendimiento moderado; ID: desprendimiento intermedio; ND: no desprendimiento.

patrones diferentes. Así, la muestra analizada es heterogénea y no corresponde al estudio de un clon aislado.

Las carbapenemasas del tipo OXA investigadas en nuestro estudio son las enzimas más prevalentes asociadas con la resistencia a carbapenémicos en A. baumannii. Aunque el OXA-23 está distribuido por todo el mundo, ninguna de nuestras cepas dio positiva para este gen ${ }^{26}$; en cambio, casi todos los aislados revelaron ser OXA-24. Este genotipo se ha descrito en brotes que causaron bacteriemias en los que las cepas permanecieron susceptibles solo a colistina y tigeciclina ${ }^{27}$ y se ha informado anteriormente en nuestra institución ${ }^{25}$. La actividad de la AZM sobre la producción y composición de biopelículas puede tener un efecto profundo que ofrezca nuevas alternativas terapéuticas especialmente para estas cepas MDR; para ello, los estudios de sinergia con la AZM y los antibióticos recomendados para el tratamiento de las infecciones por $A$. baumannii, incluidas las quinolonas y los carbapenémicos, pueden ofrecer nuevas alternativas terapéuticas.

En conclusión, una sub-MIC de AZM puede reducir la producción de biopelículas en los aislados MDR de A. baumannii con una disminución de las proteínas y el ADN en la biopelícula. Nuestros resultados son la base de estudios de sinergia que pueden ofrecer nuevas alternativas terapéuticas. 


\section{Financiamiento}

El presente estudio no recibió patrocinio, se llevó a cabo con recursos propios de la institución.

\section{Conflicto de intereses}

Los autores declaran que no existe conflicto de intereses.

\section{Responsabilidades éticas}

Protección de personas y animales. Los autores declaran que para esta investigación no se han realizado experimentos en seres humanos ni en animales.

Confidencialidad de los datos. Los autores declaran que han seguido los protocolos de su centro de trabajo sobre la publicación de datos de pacientes.

Derecho a la privacidad y consentimiento infor-

mado. Los autores declaran que en este artículo no aparecen datos de pacientes.

\section{Bibliografía}

1. Custovic A, Smajlovic J, Tihic N, Hadzic S, Ahmetagic S, Hadzagic H. Epidemiological monitoring of nosocomial infections caused by Acinetobacter baumannii. Med Arch. 2014; 68:402-6.

2. Dijkshoorn L, Nemec A, Seifert H. An increasing threat in hospitals: multidrug- resistant Acinetobacter baumannii. Nat Rev Microbiol. 2007;5:939-51.

3. Peleg AY, Seifert H, Paterson DL. Acinetobacter baumannii: emergence of a successful pathogen. Clin Microbiol Rew. 2008;21:538-82.

4. World Health Organization. 2017. Global Priority List of Antibiotic-Resistant Bacteria to Guide Research, Discovery, and Development of New Antibiotics [Internet]. World Health Organization [acceso: 10 diciembre 2018]. Disponible en: http://www.who.int/medicines/publications/WHOPPL-Short_Summary_25Feb-ET_NM_WHO.pdf

5. Longo F, Vuotto C, Donelli G. Biofilm formation in Acinetobacter baumannii. New Microbiol. 2014;37:119-27.

6. Rodriguez-Bano J, Marti S, Soto S, Fernandez-Cuenca F, Cisneros JM, Pachón J, et al. Biofilm formation in Acinetobacter baumannii: associated features and clinical implications. Clin Microbiol Infect. 2008:14:276-8.

7. Parnham MJ, Erakovic Haber V, Giamarellos-Bourboulis EJ, Perletti G Verleden GM, Vos R. Azithromycin: mechanisms of action and their relevance for clinical applications. Pharmacol Ther. 2014;143:225-45.

8. Imperi F, Leoni L, Visca P. Antivirulence activity of azithromycin in Pseudomonas aeruginosa. Front Microbiol. 2014;5:178

9. Wang A, Wang Q, Kudinha T, Xiao S, Zhuo C. Effects of fluoroquinolones and azithromycin on biofilm formation of Stenotrophomonas maltophilia. Sci Rep. 2016;6:29701.
10. Maezono $\mathrm{H}$, Noiri $\mathrm{Y}$, Asahi $\mathrm{Y}$, Yamaguchi M, Yamamoto R, Izutani N et al. Antibiofilm effects of azithromycin and erythromycin on Porphyromonas gingivalis. Antimicrob Agents Chemother. 2011;55:5887-92.

11. Chen TL, Siu LK, Wu RC, Shaio MF, Huang LY, Fung CP, et al. Comparison of one-tube multiplex PCR, automated ribotyping and intergenic spacer (ITS) sequencing for rapid identification of Acinetobacter baumannii. Clin Microbiol Infect. 2007;13:801-6.

12. Clinical and Laboratory Standards Institute (CLSI). Performance Standards for Antimicrobial Susceptibility Testing. $26^{\text {th }}$ ed. CLSI supplement M100S. Wayne, PA, USA: Clinical and Laboratory Standards Institute; 2016.

13. Magiorakos AP, Srinivasan A, Carey RB, Carmeli $Y$, Falagas ME, Giske CG, et al. Multidrug-resistant, extensively drug-resistant and pandrug-resistant bacteria: an international expert proposal for interim standard definitions for acquired resistance. Clin Microbiol Infect. 2012;18:268-81.

14. Dortet L, Poirel L, Nordmann P. Worldwide dissemination of the NDM-type carbapenemases in Gram-negative bacteria. BioMed Res Int. 2014;:2014:249856.

15. Baroud M, Dandache I, Araj GF, Wakim R, Kanj S, Kanafani Z, et al. Underlying mechanisms of carbapenem resistance in extended-spectrum beta-lactamase-producing Klebsiella pneumoniae and Escherichia coli isolates at a tertiary care centre in Lebanon: role of OXA-48 and NDM-1 carbapenemases. Int J Antimicrob Agents. 2013;41:75-9.

16. Ellington MJ, Kistler J, Livermore DM, Woodford N. Multiplex PCR for rapid detection of genes encoding acquired metallo-beta-lactamases. J Antimicrob Chemother. 2007;59:321-2.

17. Nordmann P, Poirel L. Emerging carbapenemases in Gram-negative aerobes. Clin Microbiol Infect. 2002;8:321-31.

18. Woodford N, Ellington MJ, Coelho JM, Turton JF, Ward ME, Brown S, et al. Multiplex PCR for genes encoding prevalent OXA carbapenemases in Acinetobacter spp. Int J Antimicrob Agents. 2006;27:351-3.

19. Mendoza-Olazaran S, Camacho-Ortiz A, Martinez-Resendez MF, Llaca-Diaz JM, Perez-Rodriguez E, Garza-Gon'zalez E. Influence of whole-body washing of critically ill patients with chlorhexidine on Acinetobacter baumannii isolates. Am J Infect Control. 2014:42:874-8.

20. Bou G, Cervero G, Dominguez MA, Quereda C, Martinez-Beltran J. PCR-based DNA fingerprinting (REP-PCR, AP-PCR) and pulsed-field gel electrophoresis characterization of a nosocomial outbreak caused by imipenem- and meropenem-resistant Acinetobacter baumannii. Clin Microbiol Infect. 2000;6:635-43.

21. Christensen GD, Simpson WA, Younger JJ, Baddour LM, Barrett FF, Melton DM, et al. Adherence of coagulase-negative staphylococci to plastic tissue culture plates: a quantitative model for the adherence of staphylococci to medical devices. J Clin Microbiol. 1985;22:996-1006.

22. Mendoza-Olazaran S, Morfin-Otero R, Villarreal-Trevino L, Rodriguez-Noriega E, Llaca-Diaz J, Camacho-Ortiz A, et al. Antibiotic susceptibility of biofilm cells and molecular characterisation of Staphylococcus hominis isolates from blood. PLoS One. 2015;10: e0144684.

23. Bogdan M, Drenjancevic D, Harsanji Drenjancevic I, Bedenic B, Zujic Atalic $\mathrm{V}$, talapko J, et al. In vitro effect of subminimal inhibitory concentrations of antibiotics on the biofilm formation ability of Acinetobacter baumannii clinical isolates. J Chemother. 2018;30:16-24.

24. Rapp RP. Pharmacokinetics and pharmacodynamics of intravenous and oral azithromycin: enhanced tissue activity and minimal drug interactions. Ann Pharmacother.1998;32:785-93.

25. Bocanegra-lbarias P, Peña-López C, Camacho-Ortiz A, Llaca-Díaz J, Silva-Sánchez J, Barrios H, et al. Genetic characterisation of drug resistance and clonal dynamics of Acinetobacter baumannii in a hospital setting in Mexico. Int J Antimicrob Agents. 2015;45:309-13.

26. Lee CR, Lee JH, Park M, Park KS, Bae IK, Kim YB, et al. Biology of Acinetobacter baumannii: Pathogenesis, antibiotic resistance mechanisms, and prospective treatment options. Front Cell Infect Microbiol. 2017;7:55.

27. Acosta J, Merino M, Viedma E, Poza M, Sanz F, Otero JR, et al. Multidrug-resistant Acinetobacter baumannii Harboring OXA-24 carbapenemase, Spain. Emerg Infect Dis. 2011;17:1064-7. 